\title{
Sound Source Measurement of a Semi-Circular Cylinder in a Uniform Flow by Particle Image Velocimetry
}

\author{
Yasuyuki Oguma1, Nobuyuki Fujisawa² \\ ${ }^{1}$ Department of Mechanical Engineering, Nihon University, Koriyama, Japan \\ ${ }^{2}$ Flow Visualization Research Center, Niigata University, Niigata, Japan \\ Email:oguma-y@mech.ce.nihon-u.ac.jp
}

How to cite this paper: Oguma, Y. and Fujisawa, N. (2016) Sound Source Measurement of a Semi-Circular Cylinder in a Uniform Flow by Particle Image Velocimetry. Journal of Flow Control, Measurement \& Visualization, 4, 162-170. http://dx.doi.org/10.4236/jfcmv.2016.44014

Received: August 8, 2016

Accepted: September 19, 2016

Published: October 20, 2016

Copyright $\odot 2016$ by authors and Scientific Research Publishing Inc. This work is licensed under the Creative Commons Attribution International License (CC BY 4.0). http://creativecommons.org/licenses/by/4.0/

\section{Abstract}

In this paper, the measurement of an aerodynamic sound source for a semi-circular cylinder in a uniform flow is described using Particle Image Velocimetry (PIV). This experimental technique is based on vortex sound theory, where the time derivative of vorticity is evaluated with the aid of two sets of standard PIV systems. The experimental results indicate that the sound source for the semi-circular cylinder is located around the shear layer near the edge of the semi-circular cylinder. The sound source intensity and the area are reduced in the semi-circular cylinder compared with those of a circular cylinder. This result indicates that the aerodynamic sound of the semicircular cylinder is smaller than that of the circular cylinder, which supports the microphone measurement result.

\section{Keywords}

Aerodynamic Noise, Sound Source, Semi-Circular Cylinder, Vortex Sound Theory, Particle Image Velocimetry

\section{Introduction}

The aerodynamic sound from a bluff body in a stream is an important topic of interest in fluid and environmental engineering. Many research papers have been published on the aerodynamic sound from a bluff body, such as circular and rectangular cylinders, which are summarized in review papers [1]-[3]. However, there have been fewer publications on semi-circular cylinders [4]-[6], despite the industrial importance of the design of wing mirrors for automobiles [7], Savonius wind turbine rotors [8], and so on.

Takizawa et al. [4] studied the aerodynamic characteristics of a semi-circular cylinder 
by measuring the pressure distribution on the wall surface. Later, Fujita [5] investigated the aeroacoustic characteristics of two-dimensional cylinders of various cross-sectional configurations and showed that the rounded corners in front of the bluff body are effective at improving the aeroacoustic characteristics of the bluff body. Such a body shape looks similar to the geometry of a semi-circular cylinder. More recently, Yamagata et al. [6] studied the aerodynamic characteristics of a semi-circular cylinder and showed the aerodynamic/acoustic characteristics of a semi-circular cylinder at various angles of attack. It is found that the drag forces and the Sound Pressure Level (SPL) of the semi-circular cylinder are lower than the circular cylinder at certain angles of attack; this suggests that a semi-circular cylinder has superior aerodynamic/acoustic characteristics.

In order to investigate the sound source measurements from bluff bodies, experimental methods, such as acoustic impedance [9] [10], microphone array [10] [11], cross-correlation [12]-[16], and a method based on time-resolved Particle Image Velocimetry (PIV) [17]-[20] have been reported in literature. Although these experimental methods allow the localization of the aerodynamic sound sources, the last two methods can simultaneously evaluate the sound source and velocity field, which is useful for understanding the generation mechanism of aerodynamic sound. Recently, a method using two sets of standard PIV systems has been reported for the localization of the sound source from a circular cylinder in a uniform flow [21]. This method allows the evaluation of the sound source without relying on the high-speed PIV system; thus, it is well suited for the detection of the sound source from a bluff body of complex geometry for industrial applications, though the applications have not yet been reported.

The purpose of this study is to examine the sound source distribution of a semi-circular cylinder in a uniform flow using two sets of standard PIV systems. Particular attention is placed on the visualization of the sound source distribution related to the noise production mechanism of the semi-circular cylinder by comparing it with that of the circular cylinder.

\section{Theoretical Background}

According to vortex sound theory [22] [23], the acoustic sound $p_{a}$ can be expressed by the following equation assuming a low Mach number:

$$
p_{a}=\frac{\rho x_{i}}{4 \pi c r^{2}} \iiint \frac{d}{d t}\{(\omega \times u) \cdot \nabla \varphi\} d V
$$

Here, $c$ is the speed of sound; $\varphi$ is the velocity potential; $r$ is the distance from the sound source; $t$ is time; $\boldsymbol{u}$ is the velocity vector; $V$ is the volume of interest; $x_{i}$ is the position of interest; and $\boldsymbol{\omega}$ is the vorticity vector. The vector product of vorticity and velocity in Equation (1) is called the Lamb vector, and the time derivative of the Lamb vector times the gradient of the velocity potential is the sound source intensity. In this paper, a planar PIV measurement is applied to the flow around the semi-circular cylinder, so the measurements are limited to a two-dimensional cross section perpendicular to the cylinder axis. Then, the time derivative of the Lamb vector in Equation (1) 
can be reduced to the following equation:

$$
\frac{d}{d t}\{(\omega \times u) \cdot \nabla \varphi\}=\frac{d}{d t}\left\{\left(\omega_{z} u\right) \frac{\partial \varphi}{\partial y}-\left(\omega_{z} v\right) \frac{\partial \varphi}{\partial x}\right\}
$$

Here, $\omega_{z}$ is the vorticity in $z$-direction; $u$ is the stream wise velocity; and $v$ is the normal velocity. Therefore, Equation (2) shows the sound intensity distribution of the aerodynamic sound generated from a bluff body. It should be mentioned that the velocity potential of a semi-circular cylinder is numerically obtained by solving the Laplace equation together with the Neumann boundary conditions on the wall and outer boundaries.

\section{Experimental Method}

The experiment was performed in an acoustic open-jet wind tunnel, which has been described in Ref. [21]. The test section is $190 \times 190 \mathrm{~mm}$ and $600 \mathrm{~mm}$ long. A semi-circular cylinder of $15 \mathrm{~mm}$ in diameter was placed horizontally $300 \mathrm{~mm}$ downstream of the contraction nozzle exit at the mid-height of the wind tunnel. The semi-circular cylinder was placed axisymmetric to the stream with the convex side facing upstream, as shown in Figure 1, where the sound pressure level (SPL) was found to be lower than that of the circular cylinder [6]. The experiment was performed at a mean free-stream velocity of $U_{0}=30 \mathrm{~m} / \mathrm{s}$, which corresponds to a Reynolds number of $\operatorname{Re}\left(=U_{0} d / v\right)=3 \times$ $10^{4}$, where $d$ is the diameter of the semi-circular cylinder; $U_{0}$ is the free-stream velocity; and $v$ is the kinematic viscosity of the working fluid (air). Note that the mean freestream velocity in the test section was uniform within an accuracy of $\pm 1 \%$, and the freestream turbulence level was about $1 \%$.

The time-derivative measurement of the Lamb vector in Equation (2) is performed using two sets of standard PIV systems, which are illustrated in Figure 1. Each PIV system consists of $\mathrm{Nd}$ :YAG lasers ( $50 \mathrm{~mJ} /$ pulse with 15 pulses/sec), two CCD cameras $(1280 \times 1024$ pixels with 12 bits), and a pulse generator, as described in Ref. [21].

Figure 2 shows the timing chart of the illumination and imaging for the two pairs of
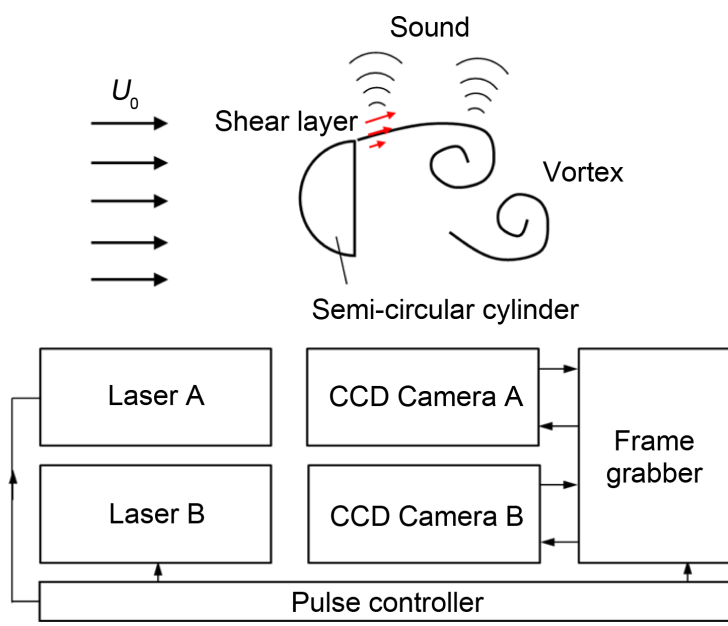

Figure 1. Experimental setup and measuring system. 


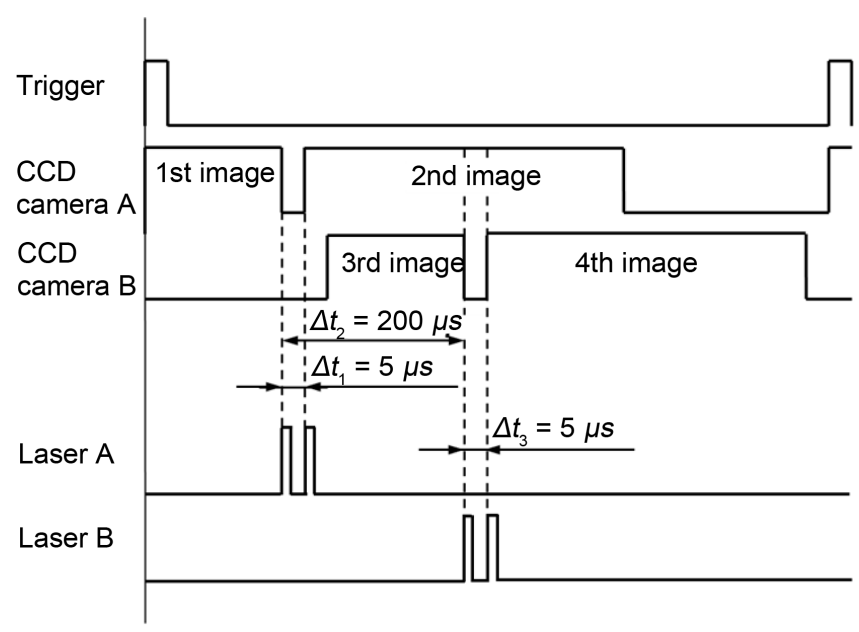

Figure 2. Timing chart of illumination and imaging.

PIV images at a short time interval. It should be mentioned that the time interval between the first and second images of camera $\mathrm{A}$ is set to $5 \mu \mathrm{sec}$, which is also the case for the third and fourth images captured by camera B. On the other hand, the time interval between the first and third images, which corresponds to the time interval between the two instantaneous velocity vectors, is set to $200 \mu \mathrm{sec}$. This time interval was determined by considering the pixel resolution of the velocity measurement, which is about 0.1 pixels in the present PIV analysis; the time interval is much shorter than the time interval of the vortex shedding $(2.5 \mathrm{msec})$. It should be mentioned that the time interval between the two cameras is set independent of the frame rate of the cameras in the present technique, while it is fixed at a constant for the time-resolved PIV system. The accuracy of time-derivative measurement of the present method can be adversely influenced by the illumination from laser B on the second image, while it does not lead to erroneous vectors in the present PIV analysis. This was confirmed by the analysis of the number of erroneous vectors.

\section{Results and Discussion}

\subsection{Sound Spectrum}

The sound spectrum of the semi-circular cylinder was measured using a microphone with a diameter of $12.7 \mathrm{~mm}$, which was located over the cylinder at a vertical distance of $500 \mathrm{~mm}$. Figure 3 shows the sound spectrum of the semi-circular cylinder in a uniform flow compared with that of a circular cylinder. It is found that the sound spectrum of the semi-circular cylinder has a peak spectrum around $400 \mathrm{~Hz}$, while that of the circular cylinder is around $370 \mathrm{~Hz}$. The Strouhal number of the semi-circular cylinder, St $\left(=f d / U_{0}\right)=0.21$, is slightly larger than that of the circular cylinder, 0.19. It is clear that the peak sound pressure level (SPL) of a semi-circular cylinder is lower than that of a circular cylinder, and the reduction effect is observed in the lower frequency range of the spectrum. These results suggest better aeroacoustic performance for a semi-circular cylinder compared with a circular cylinder. 


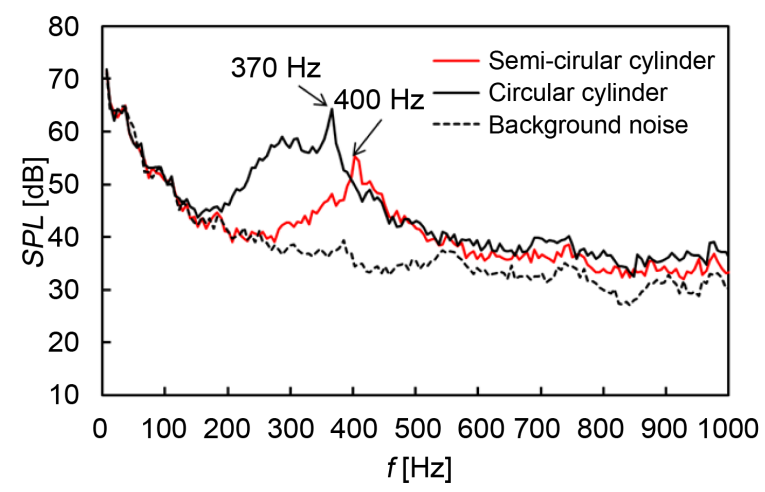

Figure 3. Frequency spectrum of aerodynamic sound.

\subsection{Mean Velocity Contour}

Figure 4(a) and Figure 4(b) show the mean velocity distribution around the semi-circular cylinder and the circular cylinder, respectively, which are obtained from the present PIV measurement. Note that the magnitude of the mean velocity is shown by a color contour. The major difference in the mean velocity field appears in the wake width downstream of each cylinder, which is narrower for the semi-circular cylinder than for the circular cylinder. It should be mentioned that the mean velocities of the shear layer from the edge of the semi-circular cylinder penetrate into the wake region and modify the wake structure such that the width and length of the wake are reduced in the semi-circular cylinder compared with the circular cylinder. Corresponding to this change, the accelerated mean velocity region over the semi-circular cylinder becomes shorter than that over the circular cylinder.

\subsection{Visualization of Sound Source Distribution}

Figure 5(a) and Figure 5(b) show the instantaneous sound source intensity distributions downstream of the semi-circular cylinder and the circular cylinder, respectively, which are evaluated from the present experimental data using Equation (2). The sound sources of the semi-circular cylinder are highly concentrated over the semi-circular cylinder and the region nearby, while those of the circular cylinder are found over the circular cylinder as well as in the near-wake region widely. This result suggests that a quantitative difference in the sound source intensity distribution is found in the semicircular cylinder and the circular cylinder. It should be noted that the instantaneous sound source intensity shows both positive and negative values due to the contribution of the signs in the vorticity $\omega_{z}$.

In order to visualize the sound source intensity distribution, the RMS sound source intensity is evaluated based on 600 instantaneous velocity fields measured at $4 \mathrm{~Hz}$ in the flow around the semi-circular cylinder. The results are compared with those of the circular cylinder, which are shown in Figure 6(a) and Figure 6(b), respectively. Although the main feature of the sound source intensity distribution of the semi-circular cylinder looks similar to that of the circular cylinder, there are quantitative differences, such as the length and width of the shear layer and the distribution in the near wake. It is found 


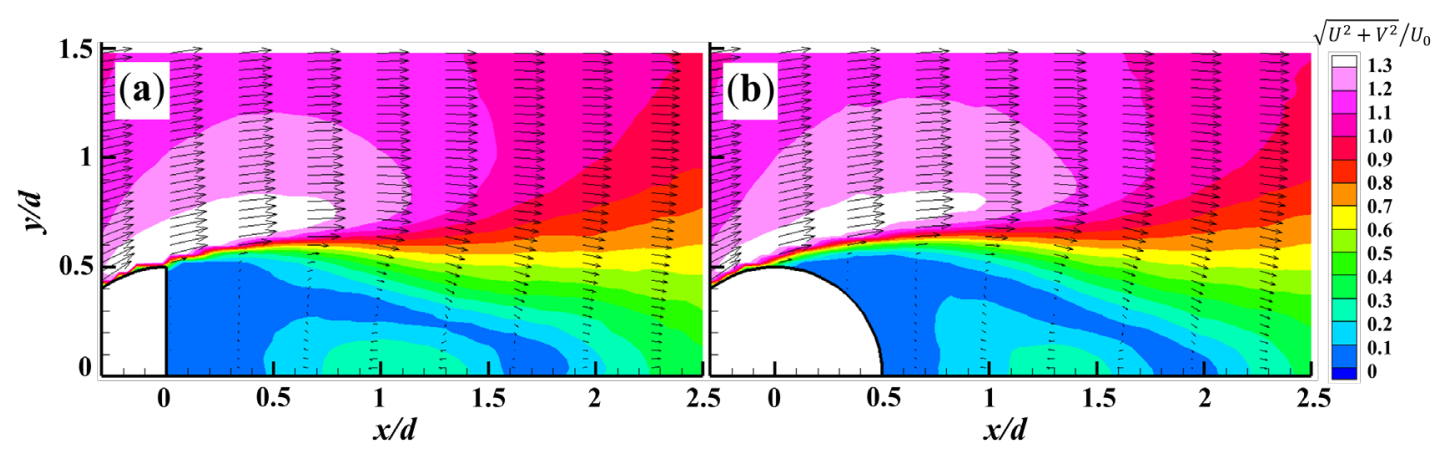

Figure 4. Mean velocity field: (a) semi-circular cylinder, (b) circular cylinder.

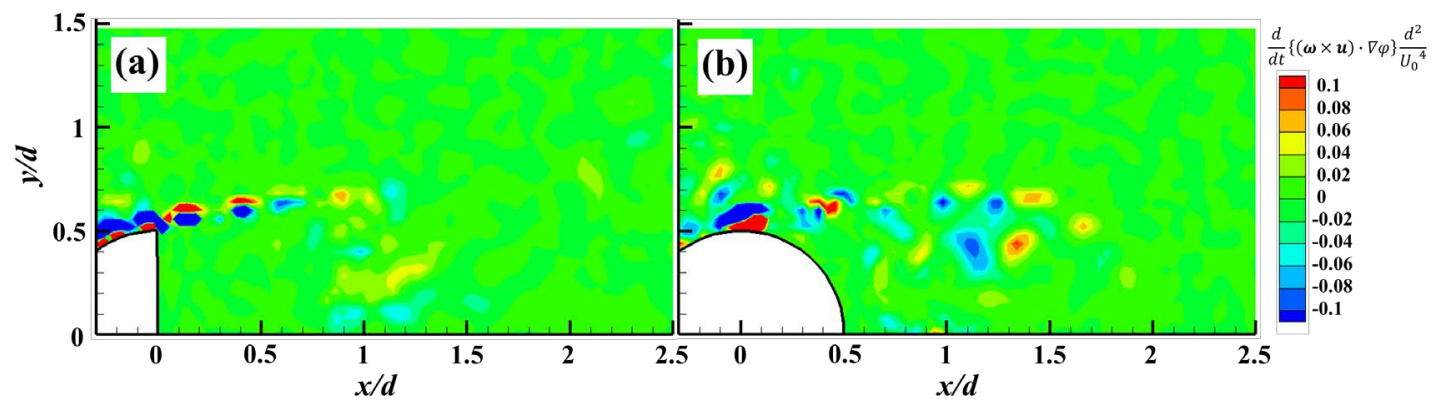

Figure 5. Instantaneous sound source intensity distribution: (a) semi-circular cylinder, (b) circular cylinder.

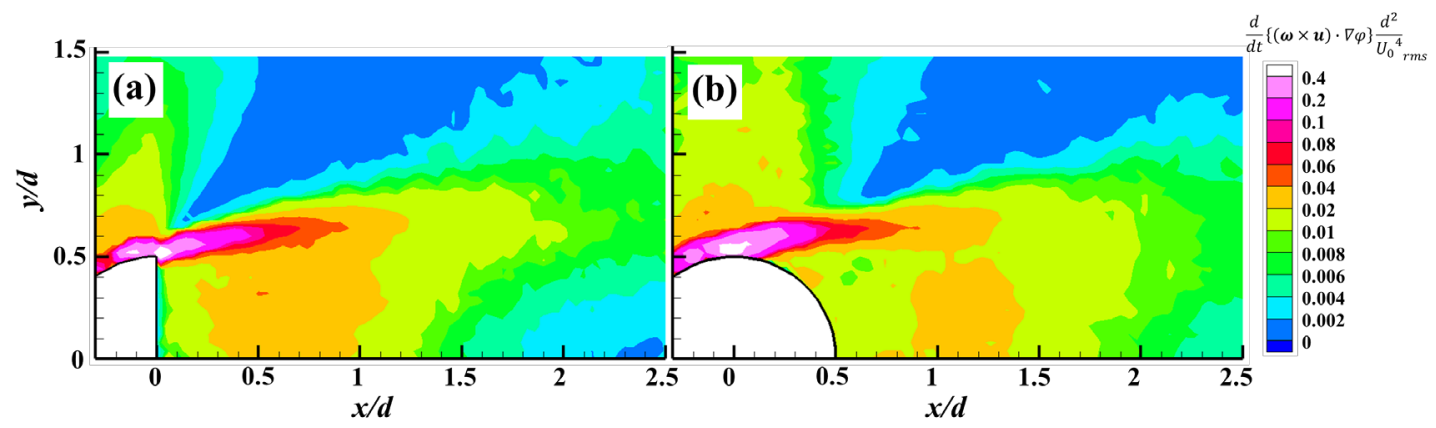

Figure 6. RMS intensity distribution of sound source: (a) semi-circular cylinder, (b) circular cylinder.

that the sound source magnitude and the area are reduced in the semi-circular cylinder compared with the circular cylinder. These changes are due to the reduction of the vortex shedding from the shear layers and the modification of vortices behind the semicircular cylinder.

\section{Conclusion}

The measurements of the velocity field and sound source intensity distribution around a semi-circular cylinder were performed using two sets of standard PIV systems, and the results were compared with those of a circular cylinder. It was found that the magnitude and area of the sound source intensity distribution in the near wake of the semi-circular cylinder were reduced compared with those of the circular cylinder. Such change in the sound source intensity distribution is due to the modification of the time 
derivatives of the vorticity and velocity potential in the near wake of the semi-circular cylinder.

\section{Acknowledgements}

The authors would like to express thanks to Prof. S. Shimizu and Prof. G. Peng of Nihon University and Dr. T. Yamagata of Niigata University for their helpful comments and discussions on this work.

\section{References}

[1] Blevins, R.D. (1984) Review of Sound Induced by Vortex Shedding from Cylinders. Journal of Sound and Vibration, 92, 455-470. http://dx.doi.org/10.1016/0022-460X(84)90191-3

[2] Blake, W.K. (1986) Mechanics of Flow-Induced Sound and Vibration. Vol. 1, General Concepts and Elementary Sources. Academic Press, Cambridge, MA, 44-64.

[3] Norberg, C. (2003) Fluctuating Lift on a Circular Cylinder: Review and New Measurements. Journal of Fluids and Structures, 17, 57-96. http://dx.doi.org/10.1016/S0889-9746(02)00099-3

[4] Takizawa, N., Okada, N. and Iwasaki, A. (1985) Wind-Tunnel Investigation of the Pressure Distribution and Deduced Characteristics of Semi-Circular Cylinder in the Vicinity of Critical Reynolds Number. Technical Report of National Aerospace Laboratory, 871, 1-44. (In Japanese)

[5] Fujita, H. (2010) The Characteristics of the Aeolian Tone Radiated from Two-Dimensional Cylinders. Fluid Dynamics Research, 42, Article ID: 015002. http://dx.doi.org/10.1088/0169-5983/42/1/015002

[6] Yamagata, T., Saito, N. and Fujisawa, N. (2016) Aeolian Tone from a Semi-Circular Cylinder in a Stream. Journal of Flow Control, Measurement \& Visualization, 4, 30-37. http://dx.doi.org/10.4236/jfcmv.2016.41003

[7] Reichl, C., Krenn, C., Mann, H. and Lang, H. (2005) Application of Numerical and Experimental Techniques for the Aero-Acoustic Characterisation of a Car Rear-View Mirror. International Journal of Aeroacoustics, 4, 185-212. http://dx.doi.org/10.1260/1475472053730020

[8] Fujisawa, N. (1992) On the Torque Mechanism of Savonius Rotors. Journal of Wind Engineering and Industrial Aerodynamics, 40, 277-292.

http://dx.doi.org/10.1016/0167-6105(92)90380-S

[9] Jones, M.G. and Stiede, P.E. (1997) Comparison of Methods for Determining Specific Acoustic Impedance. Journal of the Acoustical Society of America, 101, 2694-2704. http://dx.doi.org/10.1121/1.418558

[10] Lanslots, J., Deblauwe, F. and Janssens, K. (2010) Selecting Sound Source Localization Techniques for Industrial Applications, Sound and Vibration. 6-9 June.

[11] Bersikow, B. (1996) Experiences with Various Configurations of Microphone Arrays Used to Locate Sound Sources on Railway Trains Operated by the DB AG. Journal of Sound and Vibration, 193, 283-293. http://dx.doi.org/10.1006/jsvi.1996.0269

[12] Nakano, T., Fujisawa, N. and Lee, S. (2006) Measurement of Total-Noise Characteristics and Periodic Flow Structure around NACA0018 Airfoil. Experiments in Fluids, 40, 482490. http://dx.doi.org/10.1007/s00348-005-0089-2

[13] Nakano, T., Fujisawa, N., Oguma, Y., Takagi, Y. and Lee, S. (2007) Experimental Study on Flow and Noise Characteristics of NACA0018 Airfoil. Journal of Wind Engineering and 
Industrial Aerodynamics, 95, 511-531. http://dx.doi.org/10.1016/j.jweia.2006.11.002

[14] Henning, A., Kaepernick, K., Ehrenfried, K., Koop, L. and Dillmann, A. (2008) Investigation of Aeroacoustic Noise Generation by Simultaneous Particle Image Velocimetry and Microphone Measurements. Experiments in Fluids, 45, 1073-1085. http://dx.doi.org/10.1007/s00348-008-0528-y

[15] Nashimoto, A., Fujisawa, N., Nakano, T. and Yoda, T. (2008) Visualization of Aerodynamic Noise Source around a Rotating Fan Blade. Journal of Visualization, 11, 365-373. http://dx.doi.org/10.1007/BF03182205

[16] Oguma, Y., Yamagata, T. and Fujisawa, N. (2013) Measurement of Sound Source Distribution around a Circular Cylinder in a Uniform Flow by Combined Particle Image Velocimetry and Microphone Technique. Journal of Wind Engineering and Industrial Aerodynamics, 118, 1-11. http://dx.doi.org/10.1016/j.jweia.2013.04.003

[17] Schroeder, A., Dierksheide, U., Wolf, J., Herr, M. and Kompenhans, J. (2004) Investigation of Trailing-Edge Noise Sources by Means of High-Speed PIV. 12 th International Symposium on Applications of Laser Techniques to Fluid Mechanics, Lisbon, 12-15 July 2004.

[18] Koschatzky, V., Westerweel, J. and Boersma, B.J. (2011) A Study on the Application of Two Different Acoustic Analogies to Experimental PIV Data. Physics of Fluids, 23, Article ID: 065112. http://dx.doi.org/10.1063/1.3596730

[19] Moore, P., Lorenzoni, V. and Scarano, F. (2011) Two Techniques for PIV-Based Aeroacoustic Prediction and Their Application to a Rod-Airfoil Experiment. Experiments in Fluids, 50, 877-885. http://dx.doi.org/10.1007/s00348-010-0932-y

[20] Uda, T., Nishikawa, A., Someya, S. and Iida, A. (2011) Prediction of Aeroacoustic Sound Using the Flow Field Obtained by Time-Resolved Particle Image Velocimetry. Measurement Science and Technology, 22, Article ID: 075402. http://dx.doi.org/10.1088/0957-0233/22/7/075402

[21] Oguma, Y., Yamagata, Y. and Fujisawa, N. (2014) Measurement of Aerodynamic Sound Source around a Circular Cylinder by Particle Image Velocimetry. Journal of Flow Control Measurement and Visualization, 2, 105-109. http://dx.doi.org/10.4236/jfcmv.2014.23012

[22] Powell, A. (1964) Theory of Vortex Sound. Journal of Acoustical Society of America, 36, 177-195. http://dx.doi.org/10.1121/1.1918931

[23] Howe, M.S. (2002) Theory of Vortex Sound. Cambridge University Press, Cambridge. http://dx.doi.org/10.1017/CBO9780511755491 


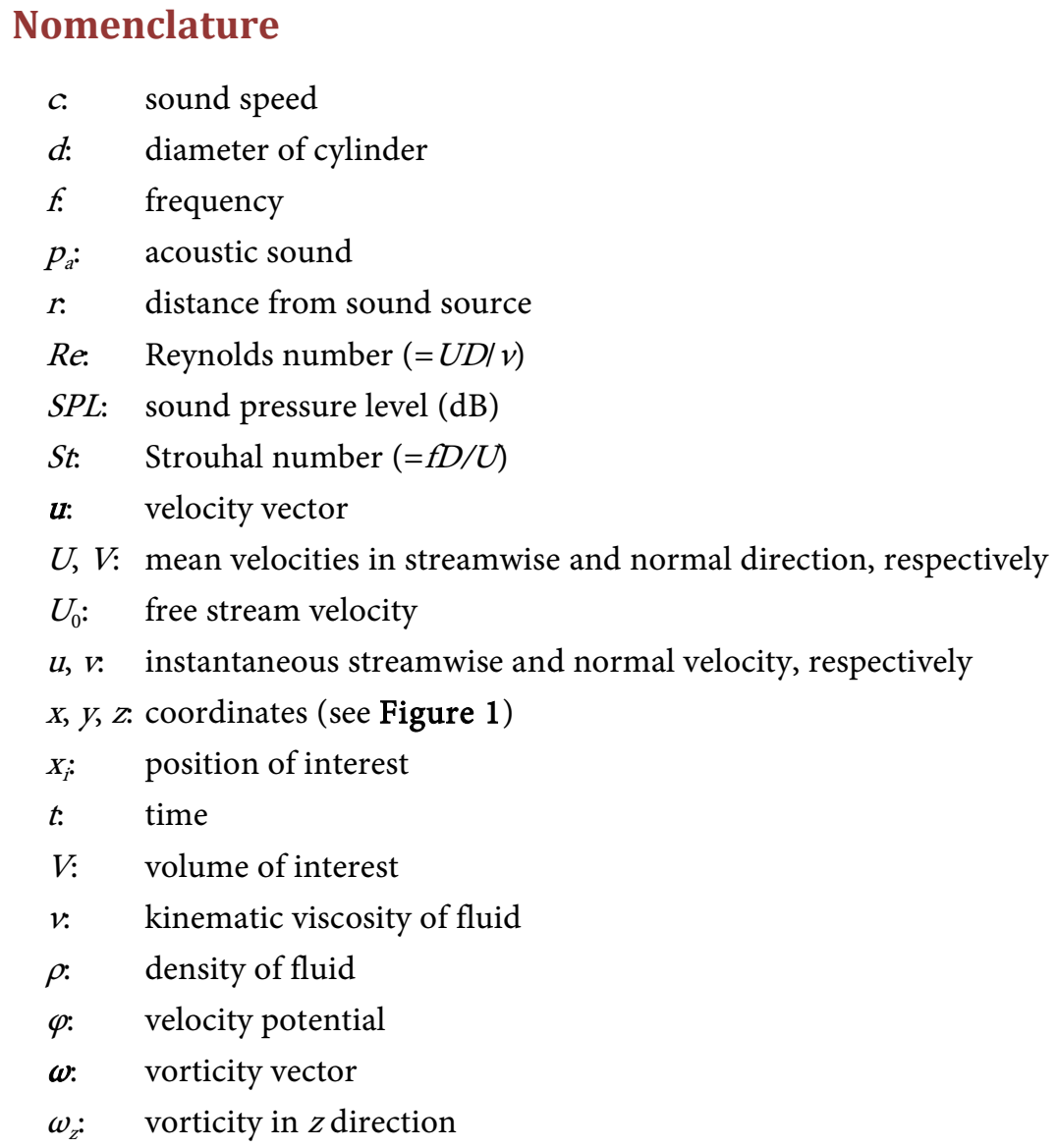

Submit or recommend next manuscript to SCIRP and we will provide best service for you:

Accepting pre-submission inquiries through Email, Facebook, LinkedIn, Twitter, etc. A wide selection of journals (inclusive of 9 subjects, more than 200 journals)

Providing 24-hour high-quality service

User-friendly online submission system

Fair and swift peer-review system

Efficient typesetting and proofreading procedure

Display of the result of downloads and visits, as well as the number of cited articles

Maximum dissemination of your research work

Submit your manuscript at: http://papersubmission.scirp.org/

Or contact jfcmv@scirp.org 